\title{
Modeling Fast Boat Motion Impact on Satellite SAR MTI Systems
}

\author{
Francisco Ceba, Eduardo Makhoul, Member, IEEE, Antoni Broquetas, Member, IEEE, \\ $\mathrm{Yu}$ Zhan and Alasdhair J. Beaton.
}

\begin{abstract}
This paper analyses the problems of imaging small and fast boats with synthetic aperture radars (SAR), which may experience severe defocusing and/or smearing. This situation is encountered, inter alia, in ocean traffic monitoring and surveillance applications where the detection of small and fast vessels is of great interest. The target modeling and impact of moving vessels in SAR images are presented and discussed using simulated results. A matched filter bank (MFB) is used before applying moving-target indication (MTI) techniques over the multichannel SAR images, refocusing the target signal in the azimuth domain and enhancing the detectability of these types of vessels.
\end{abstract}

Index Terms-Moving-target indication (MTI), Space-Borne Radar (SBR), radar detection, ocean surveillance, synthetic aperture radar (SAR), Matched Filter Bank (MFB).

\section{INTRODUCTION}

$\mathrm{T}$ HE GROWING interest in maritime traffic monitoring has created the need to design new instruments and techniques for moving-target indication (MTI) and detection purposes. In this subject, Space-Borne Radars (SBR) in Low Earth Orbits (LEO) offer the advantage of observing large areas with a reasonable operating cost [1].

Medium and large vessels typically appear as bright group of pixels in SAR images [2], which contrast with the lower clutter background. However for small boats the target signal may appear buried in clutter and noise. This is the case of rigid-hulled inflatable boats (RHIB), which can hardly be detected by SBRs, except for calm seas and slow kinematics [3]. In this paper the kinematics of small and fast ships are modeled to analyze the impact on SAR images. The goal of this paper is to evaluate the small and fast vessel detection performance of a processing chain including a MFB [9] where

Manuscript received September 16, 2014; revised October 5, 2014. Current version published March 22, 2015. This work has been supported by FPU Research Fellowship Program, Ministerio de Educación, contract AP20094590; by FI-AGAUR Research Fellowship Program, Generalitat de Catalunya, contract 2010FI EM051757; by the Spanish Ministry of Science and Innovation (MICINN) under projects TEC2011-28201-C02-01, CONSOLIDER CSD2008-00068 and TIN2014-55413-C2-1-P; and by European Commission under FP7-SPACE Project SIMTISYS Ref. 263268.

A. Broquetas, E. Makhoul,F. Ceba and Y. Zhan are with the Remote Sensing Laboratory, Department of Signal Theory and Communications, Universitat Politècnica de Catalunya-Barcelona Tech, Barcelona 08034, Spain (e-mail: broquetas@tsc.upc.edu).

A. J. Beaton is with the satellite systems and applications BU, Telespazio VEGA UK Ltd, Luton (Bedfordshire) LU1 3LU, United Kingdom (e-mail: alasdhair.beaton@vegaspace.com). each filter covers a range of chirp rates, taking into account both vessel velocity and acceleration, combined with multichannel MTI-SAR techniques [7].

\section{TARget Modeling}

\section{A. Radar Cross-Section}

To provide representative radar cross section (RCS) for the RHIB in this simulation, a 3D radar reflectivity model has been developed based on a mesh of parameterized radar scattering canonical shapes. The backscattering response of 6 canonical shapes [4] is mapped by parametric models using dominant scattering terms from geometric theory of diffraction (GTD) and physical optics (PO) solutions. Additionally to ensure solution stability over all possible viewing directions, quaternion mathematics has replaced Euler Transforms.

To model the RHIB RCS, a frame of the vessel waterline outline was constructed to meet specified dimensions $(10 \mathrm{~m}$ length $\mathrm{x} 3 \mathrm{~m}$ beam). From this frame, a computer aided design (CAD) model was created and used to identify 15 RHIB canonical elements, their dimensions and offset position (including orientation) from the body frame centroid (Fig. 1).

During the radar observation time, for every radar illumination pulse, the positions of the RHIB scattering elements are projected into radar grid using the SAR image discretization. The complex scattering of each element is calculated with the parametric models and coherently added allowing the computation of the target raw data in a pulse to pulse basis.

\section{B. Vessel kinematics}

Realistic vessel kinematics includes accelerations resulting from trajectory velocity changes and by the interaction of the vessel with the sea. This was modeled in a simulation engine that allows a vessel to alter course and speed between waypoints and a model of the interaction between the local sea characteristics and the vessel dynamical properties.

The attitude of a vessel in a seaway can be broken down into 6 elementary oscillatory motions, 3 translation motions heave, surge and sway together with 3 rotational motions yaw, pitch and roll. As an initial estimate, these motions can be

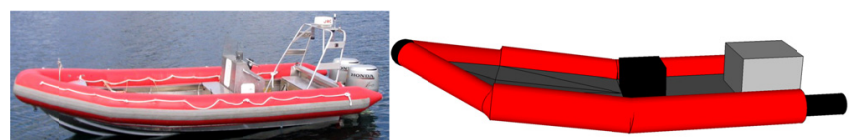

Fig. 1. RHIB Reference and CAD model. 
treated as independent modes and then coupled using the forcing provided by the incoming wave and its direction. The roll and pitch periods for a vessel can be estimated using empirical equations that base upon the vessel dimensions. Then, using wind vector parameters passed to the simulation [5], a significant wave height and period can be determined using a sea spectrum formulation [6].

With the sea and vessel dynamics characterized, a novel 6degree of freedom kinematic propagator facilitates solution of the kinematic state of the RHIB centre of gravity $(\mathrm{CoG})$ for a set of discrete time steps during the SAR image acquisition. This propagator resolves the RHIB-wave encounter frequency, the amplitudes of the 6 elementary motions and then scales these amplitudes to the RHIB using a Lorentzian function based on the modal natural frequencies. The wave elevation discrepancy between hull and CoG is solved using a 2D intersection model utilising the waterline outline and injects an additional phase offset into the final motion.

The resultant amplitude of the CoG motion can be solved as function of the maximum amplitude, the encounter frequency $\left(\omega_{\mathrm{e}}\right)$ and the wave elevation phase offset $(\varepsilon)$, for a set of time steps corresponding to the pulse repetition frequency of the SBR during the acquisition

$a(t)=a_{\max } \cos \left(\left(\omega_{e} t\right)+\varepsilon\right)$.

A geometry engine provided by Satellite Tool Kit (STK) allows solution of the scattering elements motion relative to the $\mathrm{CoG}$ using the element position parameters. Higher order kinematic terms (velocity and acceleration) are generated utilising a $3^{\text {rd }}$ order Hermite polynomial interpolation scheme. Once the kinematics are generated, the motion of each scattering element is projected into the SBR coordinates.

\section{SIgNAL MODEL}

For a monostatic SAR in X-Band working in stripmap mode, the signal captured by the antenna can be expressed using the vector notation [13-14]

$\mathbf{x}=\mathbf{s}\left(\boldsymbol{\vartheta}_{\mathbf{t}}\right)+\mathbf{c}\left(\boldsymbol{\vartheta}_{\mathbf{c}}\right)+\mathbf{n}, \mathbf{x} \in C^{M}$

as the addition of target signal $\mathbf{s}\left(\boldsymbol{\vartheta}_{\mathbf{t}}\right)$, sea clutter $\mathbf{c}\left(\boldsymbol{\vartheta}_{\mathbf{t}}\right)$ and noise $\mathbf{n}$; where $\boldsymbol{\vartheta}_{\mathbf{t}}$ and $\boldsymbol{\vartheta}_{\mathbf{c}}$ are the moving target and clutter parameter vectors respectively (range, velocities and accelerations) and $\mathbf{M}$ is the number of channels.

\section{A. Moving Target}

Let us first consider a point target with uniform accelerated rectilinear motion in the slant range-azimuth plane. Assuming a flat Earth geometry with the platform moving at $v_{p}$ with respect to ground, the range history equation during the observation time can be written as

$$
\begin{aligned}
& R(\eta)= \\
& \sqrt{\left(R_{0}+v_{s r} \eta+\frac{a_{s r}}{2} \eta^{2}\right)^{2}+\left(\left(v_{p}-v_{a z}\right) \eta+\frac{a_{a z}}{2} \eta^{2}\right)^{2}} \approx \\
& R_{0}+v_{s r} \eta+\frac{1}{2}\left[\frac{\left(v_{p}-v_{a z}\right)^{2}}{R_{0}}+a_{s r}\right] \eta^{2},
\end{aligned}
$$

being $\eta$ the azimuth time, $R_{0}$ the slant range at $\eta=0, v$ the target velocity, $a$ the target acceleration, and the subscripts $a z$ and $s r$ refer to azimuth and slant range components, respectively. The right-hand part of equation (3) corresponds to a second order Taylor series expansion around $\eta=0$.

The acquired low-pass equivalent range compressed signal in time domain with a zero-squint angle acquisition can be expressed as follows:

$$
\begin{array}{r}
S_{R C}(\tau, \eta) \approx A_{0} \cdot p_{r}\left[\tau-\frac{2 R(\eta)}{c_{0}}\right] \cdot w_{a}\left[\eta-\eta_{c}\right] \\
\cdot \exp \left\{-j \frac{4 \pi f_{0}}{c_{0}} R_{0}\right\} \cdot \exp \left\{-j \frac{4 \pi f_{0} v_{s r}}{c_{0}} \eta\right\} \\
\cdot \exp \left\{-j \frac{2 \pi f_{0}}{c_{0}}\left(\frac{\left(v_{p}-v_{a z}\right)^{2}}{R_{0}}+a_{s r}\right) \eta^{2}\right\},
\end{array}
$$

with $\tau$ representing the range time (or fast time), $p_{r}$ the range compressed pulse envelope (i.e: a sinc-like function) and $w_{a}$ the antenna weighting function (i.e: squared azimuth pattern). The second exponential term in (4) represents a shift in the Doppler spectrum of the target due to the across-track velocity component [7], which results into an azimuth displacement in the SAR image. In the case of high radial velocities the target spectrum can either fall out of the Doppler processing band or re-enter in the back-folded spectrum as an ambiguity. Furthermore they produce a high range walk not compensated by the azimuthal filters which, for high speed values, can impact in the slant range resolution. The third exponential term in (4) represents the Doppler frequency rate $\left(k_{a}\right)$, exploited for the azimuth compression [8]. As can be seen, the Doppler rate is mainly affected by $v_{a z}$ and $a_{s r}$ [7,9], producing a mismatch with the stationary world matched filter (SWMF). Therefore, the Doppler rate error can be defined as the Doppler frequency rate difference between the moving and a static target resulting in azimuth defocusing [7]:

$k_{\varepsilon}=k_{a}-k_{0}=\frac{2}{\lambda}\left(\frac{v_{a z}^{2}-2 v_{p} v_{a z}}{R_{0}}+a_{s r}\right)$,

\section{B. Clutter and noise}

The undesirable captured components are the background sea scattering $\mathbf{c}\left(\boldsymbol{\vartheta}_{\mathbf{c}}\right)$ and the thermal noise $\mathbf{n}$. In the simulation the sea clutter is modeled as a zero mean complex Gaussian process, $\mathbf{c}\left(\boldsymbol{\vartheta}_{\mathrm{c}}\right) \in \mathrm{C} \sim \mathrm{N}\left(0, \sigma_{\mathrm{c}}{ }^{2}, \mathbf{R}_{c}\right)$, where $\mathbf{R}_{c}$ represents the correlation matrix between channels. The clutter power has been obtained using the model presented in [10], which provides the normalized RCS $\sigma^{0}$ as a function of the frequency, polarization, incidence angle and sea state. Assuming this value equal for all the channels the clutter power $\sigma_{\mathrm{c}}{ }^{2}$ is then proportional to the area of the resolution 
cell. As in the TSC backscattering model [11], a wind direction correction term has been included.

Owing to the internal clutter motion (ICM) the clutter echoes backscattered from the same spatial position decorrelate between the spatially separated receivers, and this effect has been modelled incorporating a temporal covariance matrix structure, giving a correlation coefficient $\rho_{c_{n, k}}$ between channels $n$ - and $k$. A Gaussian correlation function has been assumed, which depends on the baseline time, the radar wavelength, the resolution and the wind speed [12].

The noise has been modeled as a zero-mean complex Gaussian process uncorrelated between channels, i.e., $\mathrm{n} \in \mathrm{C} \sim \mathrm{N}\left(0, \sigma_{\mathrm{n}}{ }^{2} \mathbf{I}_{M \times M}\right)$, where $\mathbf{I}_{M x M}$ represents the identity matrix. The noise power $\sigma_{\mathrm{n}}{ }^{2}$ is determined by the Noise Equivalent Sigma Zero (NESZ), a measurement of system sensitivity [15].

\section{MTI PROCESSING}

SBR systems with multiple channels aligned in the alongtrack direction provide images of the same scene acquired at slightly different positions. Once the images have been coregistered, the clutter can be cancelled and the targets dynamic parameters can be estimated. A new optimized SAR-MTI mission has been presented in [13], based on a multichannel configuration with non-uniformly displaced phase centers, using external deployable antennas. It provides improved detection capabilities for slowly and small moving vessels compared to the current state-of-the-art SAR missions. This instrument has been used throughout the simulations.

The Extended DPCA (EDPCA) algorithm, described in [14], has been implemented. An adaptive clutter cancellation jointly with a matched beam-former (to the target of interest) is performed to maximize the signal to clutter-plus-noise ratio (SCNR) at the processor's output.

In order to improve the detection performance, the MFB is used prior to MTI processing [7, 14]. Therefore, it is necessary to establish a criterion to define the number of required filters and their tuning. The proposed method to calculate the filters Doppler rates consists in measuring empirically the pulse compression losses as a function of the quadratic phase error between the received signal and the stationary world matched

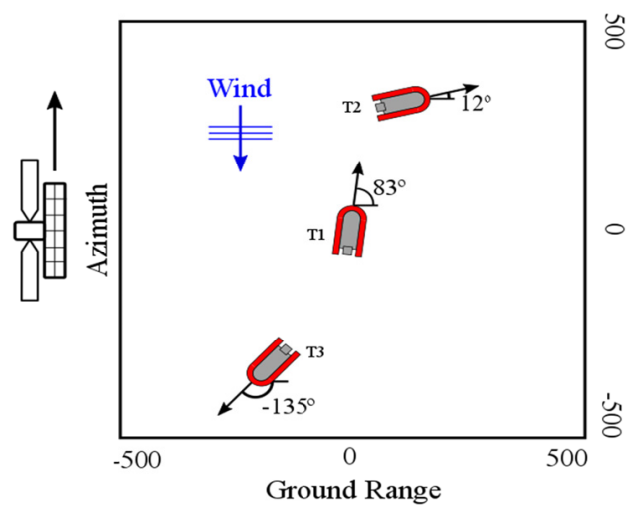

Fig. 3. Scenario layover depicting the targets location and directions. All targets are moving at 37.8 knots.

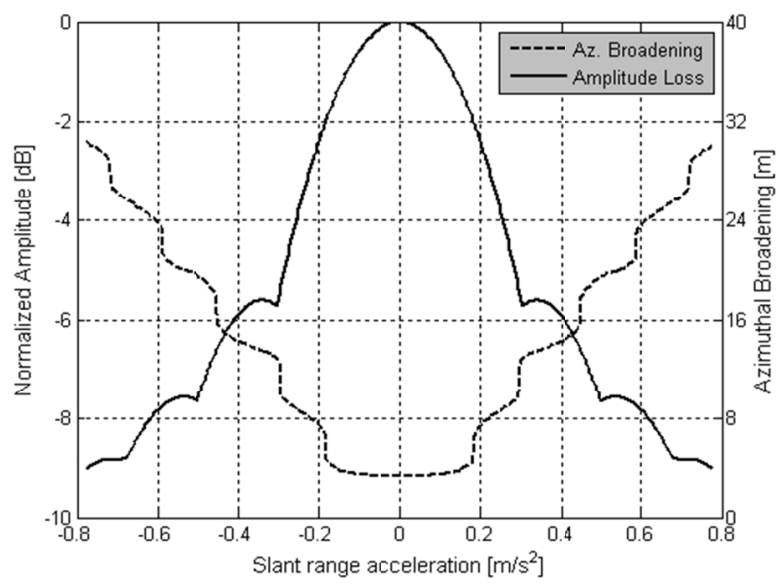

Fig. 2. Losses in amplitude (solid line) and azimuthal broadening (dashed line) as a function of slant range acceleration $a_{s r}$.

filter (SWMF) at the edges of the captured window. With this method we can predict the losses for a certain phase error with the only knowledge of the observation time and adapt each one of those filters to a certain Doppler rate interval.

The points of interest are $\phi_{\varepsilon-3 d B}=2.72 \mathrm{rad}$. and $\phi_{\varepsilon-6 d B}=5$ rad. which provide losses of $-3 \mathrm{~dB}$ and $-6 \mathrm{~dB}$ respectively. Figure 2 shows the losses and broadening in azimuth as a function of the slant range acceleration. As can be seen, an acceleration of $0.22 \mathrm{~m} / \mathrm{s}^{2}$ produces a loss of $3 \mathrm{~dB}$.

The necessary number of filters can be calculated by means of (5). For this, the $\phi_{\varepsilon-3 d B}$ point is substituted in the chirp phase formula (i.e: $\phi=\pi k \eta^{2}$ ), replacing the time $\eta$ by one half of the target observation time (namely the edge of the time window). The resulting $k_{\varepsilon}-3 d B$ value is the chirp rate distance in $\mathrm{Hz} / \mathrm{s}$ from zero to $-3 \mathrm{~dB}$ point. Then the $-3 \mathrm{~dB}$ width is obtained multiplying the previous value by 2 for spanning either sides from the center. In [9] it is mentioned that for target detection purposes, three filters are needed to cover a velocity range from -30 to $30 \mathrm{~m} / \mathrm{s}$. Nonetheless, applying the previous criterion and considering only the azimuthal velocities and typical SBR numbers in X-Band, with three filters only the range from -22.5 to $22.5 \mathrm{~m} / \mathrm{s}$ is covered. If additionally, a slant range accelerations margin from -2 to $2 \mathrm{~m} / \mathrm{s}^{2}$ is considered, the number of filters increases to seventeen. The combination of both kinematic parameters can increase the defocusing phenomenon (depending on the signs of $v_{a z}$ and $a_{s}$ ), although the across-track acceleration has a stronger impact.

From the resulting modelled kinematics it is observed that, even forcing the seaworthiness of all the vessels, the slant range acceleration within two consecutive spikes 1) is always positive and 2) never exceeds the $9 \mathrm{~m} / \mathrm{s}^{2}$ (Fig. 5). Therefore this value, jointly with the observation time shown in Table I, was used in (5) to find the relative maximum chirp rate difference with respect to the SWMF. The resulting value was divided by the filter chirp rate bandwidth to obtain a total number of 21 filters, which is the number of filters implemented in the MFB for the simulation results. 


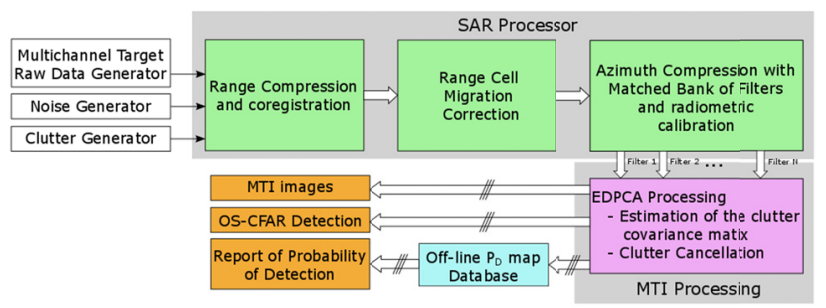

Fig. 4. Simulator breakdown structure, showing separately the SAR Processing and MTI stages. The output of the azimuthal MFB feeds the EDPCA processor, which for each filter generates a single MTI image, CFAR image and a report of the $\mathrm{P}_{\mathrm{D}}$ for each target.

\section{Simulation Results}

The structure of the SAR processor and MTI block are depicted in Fig. 4. Despite of generating the clutter and noise at SAR image level, their raw data have been obtained by inverse SWMF SAR processing in order to account for the impact of limited SAR resolution and azimuth focusing with different chirp rates in the MFB. Black arrows represents multichannel images for a single layer (i.e: target signal, clutter or noise) while the white arrows are the combination of the above (multichannel and multilayer). The arrows striped with three lines are image streams of multiple filter outputs with multiple layers (the multichannel domain has been combined to form the EDPCA image).

The simulations have been carried out with the parameters shown in Table I. The scenario contains three vessels, all of them moving at 37.8 knots in the slant range-azimuth plane but with different directions (see Fig. 3), resulting in different kinematics for each vessel. In order to make a fair comparison, all the RHIBs have been modelled with the same maximum RCS. As can be seen in Fig. 5 the hull of the boats experiences strong impacts against the water which produces the spikes in the slant range acceleration profile. The processing of these acceleration peaks with a MFB using constant Doppler rates results into a severely defocused image (with the best filter a loss of $-10 \mathrm{~dB}$ was obtained); thus a SAR observation interval excluding these strong acceleration peaks has been selected in the simulations for all boats (shadowed area of Fig. 5). Target T2 is moving with a $v_{a z}=8.17 \mathrm{~m} / \mathrm{s}$ and $a_{s r}=1.58 \mathrm{~m} / \mathrm{s}^{2}$, such that a filter tuned to $5343.8 \mathrm{~Hz} / \mathrm{s}$ is expected to refocus it. Target T3 with $v_{a z}=-25 \mathrm{~m} / \mathrm{s}$ and $a_{s r}=0.87 \mathrm{~m} / \mathrm{s}^{2}$, is best refocused also with a filter tuned to $5343.8 \mathrm{~Hz} / \mathrm{s}$. Target T1 moves at $v_{a z}=35$ $\mathrm{m} / \mathrm{s}$ and $a_{s r}=7.5 \mathrm{~m} / \mathrm{s}^{2}$ and it is foreseen to be properly focused

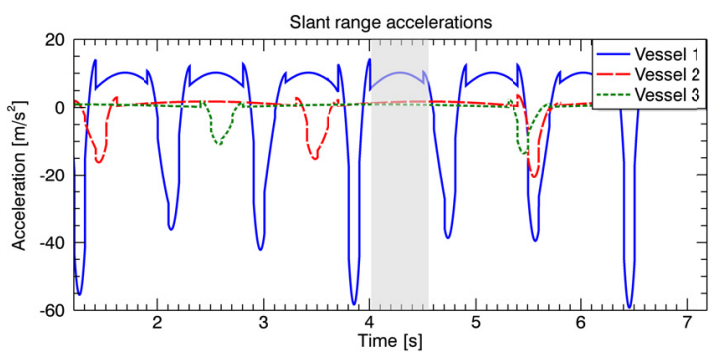

Fig. 5. Accelerations profiles for the three vessels in the slant range coordinate. The shadowed area represents the time window within which the SAR is acquiring data.
TABLE I

SCENARIO AND RADAR PARAMETERS

\begin{tabular}{llc}
\hline \hline Symbol & \multicolumn{1}{c}{ Parameter } & Value \\
\hline$R_{0}$ & Slant range to the target at $\eta=0$ & $6 \cdot 10^{5} \mathrm{~m}$ \\
$V_{p}$ & Platform velocity & $7000 \mathrm{~m} / \mathrm{s}$ \\
$\lambda$ & Radar wavelength & $0.031 \mathrm{~m}$ \\
$P R F$ & Pulse repetition frequency & $4183 \mathrm{~Hz}$ \\
$B W_{r}$ & Range processing bandwidth & $62.7 \mathrm{MHz}$ \\
$B W_{a}$ & Azimuth processing bandwidth & $2.6 \mathrm{KHz}$ \\
$T_{o b s}$ & Observation time & $0.49 \mathrm{~s}$ \\
$v_{w}$ & Wind velocity & $8.25 \mathrm{~m} / \mathrm{s}$ \\
$\theta_{w}$ & Wind direction ${ }^{\text {a }}$ & $-90^{\circ}$ \\
$H_{l / 3}$ & Significant wave height & $1.802 \mathrm{~m}$ \\
$T_{w}$ & Wave encountered period & $2.66 \mathrm{~s}$ \\
$N E S Z$ & Noise Equivalent Sigma Zero & $-23 \mathrm{~dB}$ \\
$\sigma^{0}$ & Clutter sigma-zero & $-17.5 \mathrm{~dB}$ \\
$\tau^{c}$ & Clutter correlation time & $11 \mathrm{~ms}$ \\
$P o l$ & Instrument polarization & $\mathrm{HH}$ \\
$R C S$ & Maximum RCS & $2 \mathrm{dBsm}$ \\
\hline \hline
\end{tabular}

${ }^{\mathrm{a}}$ Relative to radar look direction.

${ }^{\mathrm{b}}$ For all the targets.

by a MFB set to $5703.4 \mathrm{~Hz} / \mathrm{s}$. In practice one of the adjacent filters may provide better response than the expected matched filter, owing to the fact that kinematics are time dependent. This is the case of targets T1 and T3, for which filters tuned to $5321.3 \mathrm{~Hz} / \mathrm{s}$ and $5680.9 \mathrm{~Hz} / \mathrm{s}$ give an increment of $2.3 \mathrm{~dB}$ and $1.7 \mathrm{~dB}$ respectively, compared with the expected matched filters. Compared with the SWMF the improvement factors are $9.2 \mathrm{~dB}$ for vessel $\mathrm{T} 3$ and $13.5 \mathrm{~dB}$ for vessel $\mathrm{T} 1$. Special attention has been paid to targets $\mathrm{T} 2$ and $\mathrm{T} 3$, since their high radial velocities produce high mismatch with respect to the nominal range cell migration curvature, resulting in slant range defocusing [7]. Moreover for target T2 the high slant range velocity causes a back-folding in the Doppler spectrum, producing its breakup into two halves located at the edges of the spectral domain.

For a duly assessment high across-track velocities shall be considered in order to correct the range cell migration suffered. Throughout the simulations, the results obtained with and without the tailored range cell migration correction (RCMC) have been compared. For target T1 a static RCMC has been applied since the differences can be neglected. For target T2 and T3 adapting the RCMC to each filter of the bank provided a gain of $4 \mathrm{~dB}$ and $9.1 \mathrm{~dB}$ respectively referred to the stationary RCMC reconstruction.

With the SWMF, the maximum amplitudes of the boats were at $-10 \mathrm{dBsm}$. A minimum gain of $8 \mathrm{~dB}$ is obtained for all of them with the best matched filter using the presented architecture, obtaining a probability of detection after the MTI processing that exceeds the $90 \%$ in all three cases. A probability of false alarm $\left(P_{f a}\right)$ of $10^{-5}$ has been assumed. Figure 6 shows the EDPCA images and the detection maps at the output of a constant false alarm rate (CFAR) detector. The EDPCA response is velocity dependent [13] and that is why the corresponding processor's outputs is different for each adapted target processing, i.e., T1, with the smallest radial velocity, has reduced SCNR conditions compared to T2 and T3. 

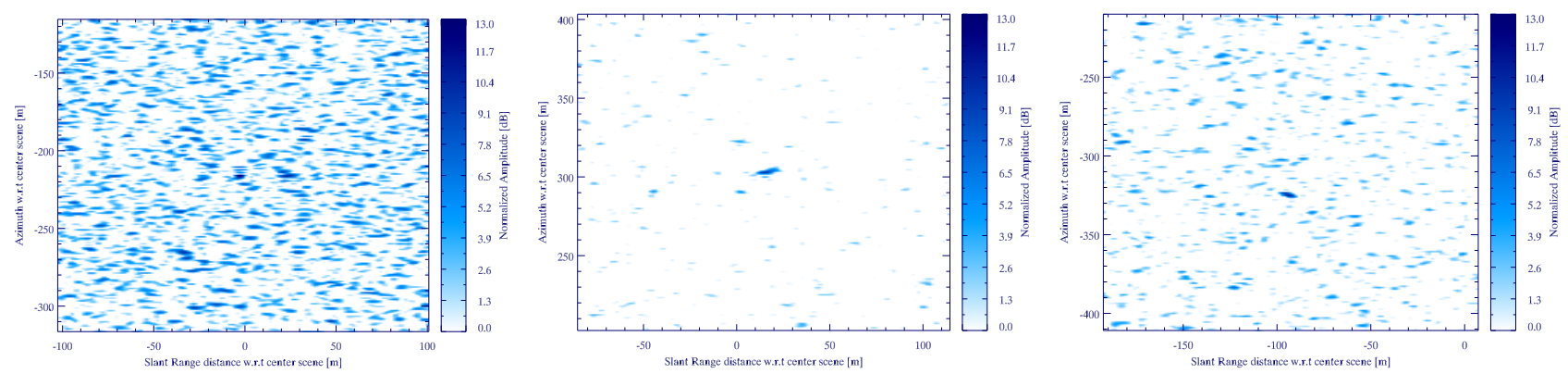

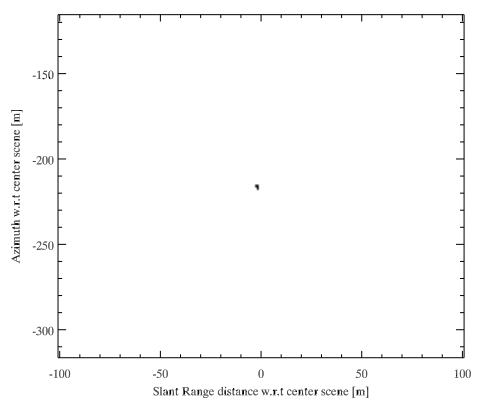

(a)

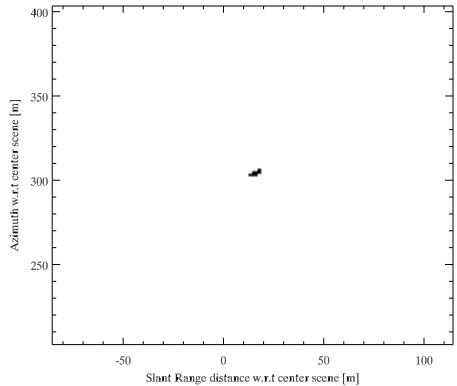

(b)

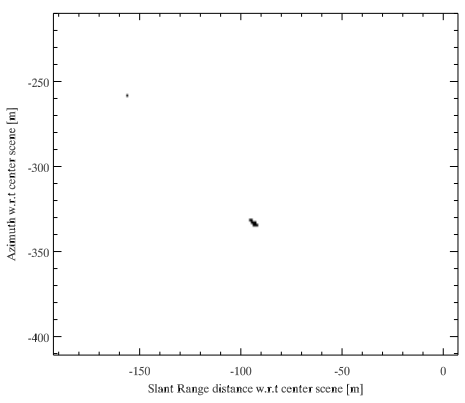

(c)

Fig. 6. EDPCA images (first row) and CFAR products (second row) applying a $\mathrm{P}_{\mathrm{fa}}=10^{-5}$. Each column represents the results for (a) target 1 , (b) target 2 and (c) target 3 , obtained with their respective best-matched filters.

\section{CONCLUSIONS}

In this paper the radar scattering and kinematics for a small and fast boat sailing with a strong breeze has been modelled, considering realistic accelerations. To increase the SCNR and SAR detection performance a MFB has been included before the MTI processing stage. This architecture combined with the proposed SAR-GMTI mission [13] has been shown to be able to detect a RHIB experiencing moderate accelerations with three different heading directions.

When the high acceleration spike in slant range is encountered within the radar observation time, the proposed processing chain is not able to detect the vessels. For the simulated scenario, the three analyzed vessels experienced different peak acceleration periods due to differences in their kinematics and headings with respect to wind direction. Assuming a uniformly distributed random location of the time observation window with respect to each vessel acceleration profile in Fig. 5 the probability of observing the target free of acceleration peaks can be obtained. Averaging the 3 considered cases the probability of observing the fast boats in the simulated sea state without the acceleration peak is estimated to be $43 \%$. To overcome this limitation, higher order terms could be considered in the range history in equation (3), to model and compensate the high target dynamics. However this would result into a higher number of degrees of freedom and larger number of filters in the MFB.

\section{REFERENCES}

[1] Skolnik M.,"Spaceborne radar for the global surveillance of ships over the ocean", Proceedings of the 1997 National Radar Conference, pp.120 $-125,1997$.
[2] Jackson, Christopher R., et al. "Synthetic aperture radar marine user's manual" US Department of Commerce, 2004.

[3] Blake, T. M. "The detection and tracking of small fast boats using HF surface wave radar" HF Radio Systems and Techniques, IEE Conference Publication, pp. 219-224, 2000.

[4] Jackson, Julie A. "Three-Dimensional Feature Models for Synthetic Aperture Radar and Experiments in Feature Extraction" Electronic Thesis. Ohio State University, 2009.

[5] Doerry, A. W. "Ship Dynamics for Maritime ISAR Imaging" Sandia National Laboratories, U.S. 2008

[6] Walter H. Michel, "Sea spectra simplified, "Marine Technology, 1968, Vol. 5, No. 1, pp. 17-30.

[7] Cristallini, D., Pastina, D., Colone, F., Lombardo, P. "Efficient detection and imaging of moving targets in SAR images based on chirp scaling,", IEEE Transactions on Geoscience and Remote Sensing, 2013, vol. 51, no 4 , pp. 2403-2416

[8] Ian G. Cumming, Frank H. Wong, "Digital Processing of Synthetic Aperture Radar Data: Algorithms and Implementations", Artech House, Norwood (MA), Jan. 2005.

[9] Charles E. Livingstone, Alan A. Thompson,"The moving object detection experiment on RADARSAT-2,"Can. J, Remote Sensing,vol. 30, N o. 3, pp.355-368, 2004.

[10] V. Gregers-Hansen, R. Mital, “An improved empirical model for radar sea clutter reflectivity, "IEEE Trans. Aerosp. Elect. Sys., vol. 48, N o . 4 , pp.3512-3524, 2012.

[11] I. Antipov,"Simulation of Sea Clutter Returns," DSTO Electronic and Surveillance Research Laboratory,Salisbury,DSTO-TR-0679, Jun. 1998.

[12] S. Frasier, A. Camps, "Dual-Beam Interferometry for Ocean Surface Current Vector Mapping, "IEEE Trans. Aerosp. Elect.Sys., vol. 39, N o . 2 , pp.401-414, Feb. 2001.

[13] E. Makhoul, A. Broquetas, J. Ruiz-Rodon, Y. Zhan, and F. Ceba, "A Performance Evaluation of SAR-GMTI Missions for Maritime Applications," IEEE Transactions on Geoscience and Remote Sensing, vol. 53, no. 5, pp. 2496-2509, May 2015.

[14] D. Cerutti-Maori, I. Sikaneta, "A Generalization of DPCA Processing for Multichannel SAR/GMTI Radars," IEEE Trans. Geosc. Remote Sens., vol. 51, No. 1, pp. 560-572, Jan. 2013.

[15] Curlander, John C., McDonough, Robert N., "Synthetic aperture radar”, New York, Wiley, 1991. 